\begin{tabular}{|c|l|}
\hline Title & Selenium-Ioaded zeolite (ZSM-5): I. Structures and fundamental properties \\
\hline Author(s) & Saitoh, A kira; Tanaka, Keiji \\
\hline Citation & $\begin{array}{l}\text { Journal of A pplied Physics, 105(11), 113530 } \\
\text { https://doi.org/10.1063/1.3143609 }\end{array}$ \\
\hline Issue Date & 2009-06-01 \\
\hline Doc URL & http://hdl.handle.net/2115/38778 \\
\hline Rights & $\begin{array}{l}\text { Copyright 2009 A merican Institute of Physics. This article may be downloaded for personal use only. A ny other use } \\
\text { requires prior permission of the author and the A merican Institute of Physics. }\end{array}$ \\
\hline Type & article \\
\hline File Information & 105-11_113530.pdf \\
\hline
\end{tabular}

Instructions for use 


\title{
Selenium-loaded zeolite (ZSM-5): I. Structures and fundamental properties
}

\author{
Akira Saitoh and Keiji Tanaka ${ }^{a)}$ \\ Department of Applied Physics, Graduate School of Engineering, Hokkaido University, Sapporo 060-8628, \\ Japan
}

(Received 19 March 2009; accepted 4 May 2009; published online 11 June 2009)

\begin{abstract}
Atomic structures of Se clusters impregnated into ZSM-5 single crystals have been investigated through structural and optical experiments. Raman-scattering spectroscopy suggests that Se in the zeolite takes single chain structures. X-ray diffraction implies that the chains form fourfold (not threefold as in trigonal Se) helices. The chain length is estimated from weight and electron-spin resonance measurements at $10^{2}$ atoms. The material shows an optical absorption edge at $\hbar_{\omega}$ $\simeq 2.2 \mathrm{eV}$, which accompanies dichroism, suggesting chain alignment in the zeolite pores. (C) 2009 American Institute of Physics. [DOI: 10.1063/1.3143609]
\end{abstract}

\section{INTRODUCTION}

The nanostructured chalcogenide glass has attracted considerable interest due to covalent and semiconductor properties. ${ }^{1}$ For instance, two-dimensional nanostructures including ultrathin and multilayer films are demonstrated to have peculiar thermal and optical properties, being different from those of the corresponding bulk glasses. ${ }^{1-3}$ Threedimensional nanostructures, which can be obtained, for instance, in heterogeneous oxychalcogenide glasses such as $\mathrm{GeO}_{2}-\mathrm{GeS}_{2}$, also exhibit marked optical and photostructural properties. ${ }^{1,4}$ On the other hand, studies on zero- and onedimensional nanostructured chalcogenides have relatively been limited. ${ }^{1}$

A notable exception is to use the zeolite, ${ }^{5}$ a variety of porous $\mathrm{Si}-\mathrm{Al}-\mathrm{O}$ crystals, and similar materials as hosts for incorporating low-dimensional chalcogen clusters. In pioneering studies (1976 ), Bogomolov and co-workers in Ioffe Institute discovered $\mathrm{X}$-ray irradiation effects in S-loaded $\mathrm{Na} A,{ }^{6}$ quantized optical spectra in Se-loaded $\mathrm{Na} X,{ }^{7}$ and anisotropic optical absorptions in Se- and Te-loaded $\mathrm{Na} M$ (natural mordenite). ${ }^{8}$ Valuable studies including photoinduced phenomena and theoretical calculations have also been reported from other groups. ${ }^{9-34}$ These results suggest or demonstrate that the chalcogen-loaded zeolite, in which the chalcogen works as a dye in organic polymers, is promising for optical applications. However, in some studies, powderlike zeolites have been employed, which may exhibit substantial surface effects. ${ }^{18,28,34}$ In addition, there is a variety of zeolites with pore shapes and covalencies, ${ }^{5}$ while most of previous studies have employed zeolites containing ionic pores, ${ }^{15-27,29-34}$ which may mask covalent properties of chalcogens. Accordingly, further work relating atomic structures with macroscopic properties in chalcogen-loaded covalent zeolites seems intriguing.

We here study structural properties of Se-loaded ZSM-5 zeolite $(z-\mathrm{Se})$ with a motivation for producing new photonic devices. The reasons why we select this system are, at least, three. First, we can obtain transparent and submillimeter size

\footnotetext{
${ }^{a)}$ Author to whom correspondence should be addressed. Electronic mail: keiji@eng.hokudai.ac.jp.
}

ZSM-5, ${ }^{35-37}$ which is suitable to single-crystalline, structural, and optical experiments. Second, atomic structures of ZSM-5 are appropriate. As schematically illustrated in Fig. 1, it has two kinds of pores, ${ }^{36,37}$ one being parallel to the crystalline $b$ axis and the other being zig-zag on the $a-c$ plane. Diameters of these pores are approximately the same, with framework and free (taking oxygen diameter into account) diameters of $\sim 0.83$ and $\sim 0.56 \mathrm{~nm}$. Moreover, ZSM-5 is fairly covalent with the $\mathrm{Al} / \mathrm{Si}$ ratio of $\sim 0.02$ (Refs. 36 and 37 ) so that ionic interaction between loaded $\mathrm{Se}$ and pore walls may be the least. Lastly, a comparative study on S, Se, and Te has suggested that only Se can be incorporated with substantial amounts into this zeolite. ${ }^{38}$

\section{EXPERIMENTS}

Se-loaded samples were prepared as follows. Singlecrystalline ZSM-5 with a typical dimension of about 40

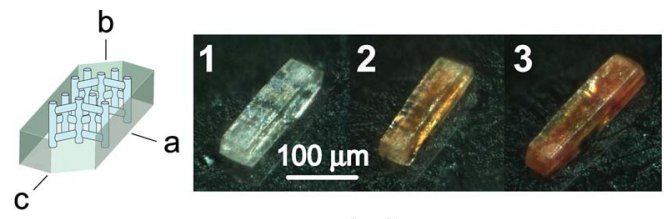

(a)

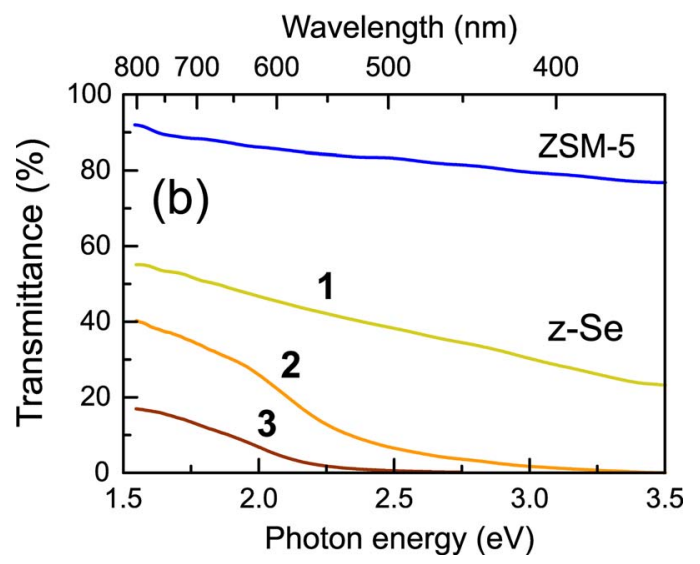

FIG. 1. (Color online) (a) A schematic illustration of a ZSM-5 crystal (left) with crystalline axes and exaggerated pores and photographs of three $z$-Se samples viewed from the $a$ surface and (b) transmission spectra of a pure zeolite (top) and the three samples. 
$\times 40 \times 200 \mu \mathrm{m}^{3}$ was prepared by Kiyozumi, the procedure being similar to that described in Ref. 37 . The ZSM-5 crystals had contained organic templates, which were desorbed by a heat treatment at $500{ }^{\circ} \mathrm{C}$ for $3 \mathrm{~h}$ in vacuum just before chalcogen loading. The desorption was confirmed through disappearance of infrared absorption peaks of the template. ${ }^{38}$ Then, a pile of ZSM-5 $(\sim 5 \mathrm{mg} \simeq 5000$ pieces $)$ and Se chunks with six-nine purity were vacuum-sealed in a doublearms quartz ampoule, which was heated so that the chalcogen was impregnated from vapor phases into the zeolite. The temperature and duration, which were selected after several trials for producing dense-colored samples, were $300{ }^{\circ} \mathrm{C}$ and $\sim 3 \mathrm{~h}$ (the melting temperature of Se is $217^{\circ} \mathrm{C}$ ), where the zeolite pile is kept at $\sim 150{ }^{\circ} \mathrm{C}$. As reported previously, ${ }^{37,38}$ these thermal treatments should be performed under slow $\left(<30{ }^{\circ} \mathrm{C} / \mathrm{h}\right)$ heating and cooling for suppressing formation of cracks, which made the zeolite smoggy. Prepared $z$-Se was fairly stable, with no color change at least for 1 year under storage in dry atmosphere. However, a sample stored for 8 months after Se loading presented infrared peaks due to $\mathrm{H}_{2} \mathrm{O}$. For subsidiary measurements, amorphous $\mathrm{Se}(a-\mathrm{Se})$ films were prepared through vacuum evaporation of the $\mathrm{Se}$ chunks onto glass substrates held at room temperature. Polycrystalline trigonal $\mathrm{Se}(t$-Se) films were obtained through thermal crystallization of the amorphous films.

Structures and properties were inspected at five points. First, an amount of loaded Se was estimated from measurements of weight changes upon thermal desorption of Se, the detail being described later. Second, Raman-scattering spectroscopy was performed using a triple-spectrometer system (Jobin Yvon, T64000) equipped with a cooled chargecoupled device. Probe light was obtained from linearly polarized $\mathrm{Ar}^{+}(488$ and $515 \mathrm{~nm})$ and $\mathrm{He}-\mathrm{Ne}(633 \mathrm{~nm})$ lasers, which provided focused light spots with a diameter of $\sim 1 \mu \mathrm{m}$ and an intensity of $\sim 50 \mu \mathrm{W}$. Third, $\mathrm{x}$-ray diffraction patterns were obtained using a $\mathrm{Cu}$ rotating anode (Rigaku, Ru-300), a conventional diffractometer, and a proportional counter having a graphite monochromator. Samples, consisting of several hundred $z$-Se pieces, which were fixed to thin $(\sim 30 \mu \mathrm{m})$ sheets of highly oriented pyrolytic graphite, were inspected in a Laue (transmission) arrangement for suppressing intense peaks arising from the graphite. Fourth, electron spin resonance (ESR) spectra were monitored using an X-band continuous-wave spectrometer (JEOL, FE1X) at 80 and $290 \mathrm{~K}$. Concentrations of unpaired electrons were estimated by using $\mathrm{CuSO}_{4} \cdot 5 \mathrm{H}_{2} \mathrm{O}$ as a standard. Lastly, visible-range transmission spectra were obtained for single samples, which were put on an aperture with a diameter of $25 \mu \mathrm{m}$, using a double-beam spectrometer (JASCO, V570) at 80 and 293 K. Dichroic spectra were also obtained using the spectrometer and polarizers. Nevertheless, the transmittance was largely affected by light scattering. Accordingly, we also employed another single-beam optical arrangement using lasers and photodetectors, which could measure the intensities of transmitted and forwardscattered light.

\section{RESULTS}

\section{A. Color variation}

Figure 1 shows overall color variations in $z$-Se with the corresponding transmission spectra. ZSM-5 is fairly transparent in the visible region, while Fig. 1(b) shows a small transmittance decrease at short wavelengths, which probably reflect light scattering. Se loading has added color to the zeolite. However, despite repeated trials with varied preparation conditions, $z$-Se samples produced under a single batch reaction have appeared in different colors, as exemplified in Figs. 1(a) and 1(b). The transparent zeolite has changed to pale yellow, orange, and brown, the number ratio being roughly 1:6:3. Moreover, single samples possess spatial color variations. These results may imply some imperfections in the present ZSM-5 crystals. Since the brown sample gives the most intense Raman-scattering peak attributable to Se, we focus on the brown in the following.

\section{B. Loaded Se density}

Quantities of loaded Se have been determined through measuring weight loss upon thermal desorption. Measurements were done three times for $\sim 3000$ pieces of brown $z$-Se with total weights of $2.8-3.0 \mathrm{mg}$, which became transparent with weights of $2.0-2.3 \mathrm{mg}$ after a heat treatment at $250{ }^{\circ} \mathrm{C}$ for $20 \mathrm{~min}$. (The same heat treatment gave no weight changes for pure zeolites.) The weight loss of $0.5-1 \mathrm{mg}$ corresponds to $\sim 10^{15} \mathrm{Se}$ atoms per a ZSM-5 crystal. On the other hand, the total length of pores in the ZSM-5 is estimated from the crystal structure ${ }^{37}$ at $10^{6} \mathrm{~m} /$ piece. Accordingly, assuming that the $10^{15} \mathrm{Se}$ atoms take helical chain structures with a helix pitch of $\sim 0.6 \mathrm{~nm}$ (Table I), we envisage that $\sim 100 \%$ pores are filled with $\mathrm{Se}$ atoms in the brown sample.

\section{Raman scattering}

Figure 2 shows Raman-scattering spectra of $z$-Se, $a$-Se, and $t$-Se. The spectra are obtained using linearly polarized laser light, while the polarization of scattered light is unresolved. Note that ZSM-5 has given no Raman-scattering peaks under the present measuring condition. We see in Fig. 2 that some peaks appear on flat or oblique baselines. $z$-Se inspected under the electric field $E$ of probe light parallel to the zeolite $c$ axis $(E \| c), a$-Se, and $t$-Se show relatively sharp Raman-scattering peaks with flat baselines. On the other hand, in all $z$-Se with $E$ not parallel to the $c$ axis, the baselines increase with the scattering wavenumber, which is attributable to photoluminescence, the details being reported in Ref. 38. The main Raman peak is located at $\sim 240 \mathrm{~cm}^{-1}$ in $t$-Se, $\sim 250 \mathrm{~cm}^{-1}$ in $a$-Se, both being consistent with previous results, ${ }^{39}$ and $\sim 260 \mathrm{~cm}^{-1}$ in $z$-Se for all the $E$ directions. In addition, $z$-Se presents small humps at $\sim 380$ and $\sim 510 \mathrm{~cm}^{-1}$, the intensities varying from sample-to-sample and with measuring conditions. The $380 \mathrm{~cm}^{-1}$ peak will be discussed later, while it is straightforward to identify the $510 \mathrm{~cm}^{-1}$ peak as an overtone of the $260 \mathrm{~cm}^{-1}{ }^{12}$ It should be mentioned that no peaks arising from other species such as $\mathrm{O}_{2}\left(1128 \mathrm{~cm}^{-1}\right)$ were detected. 


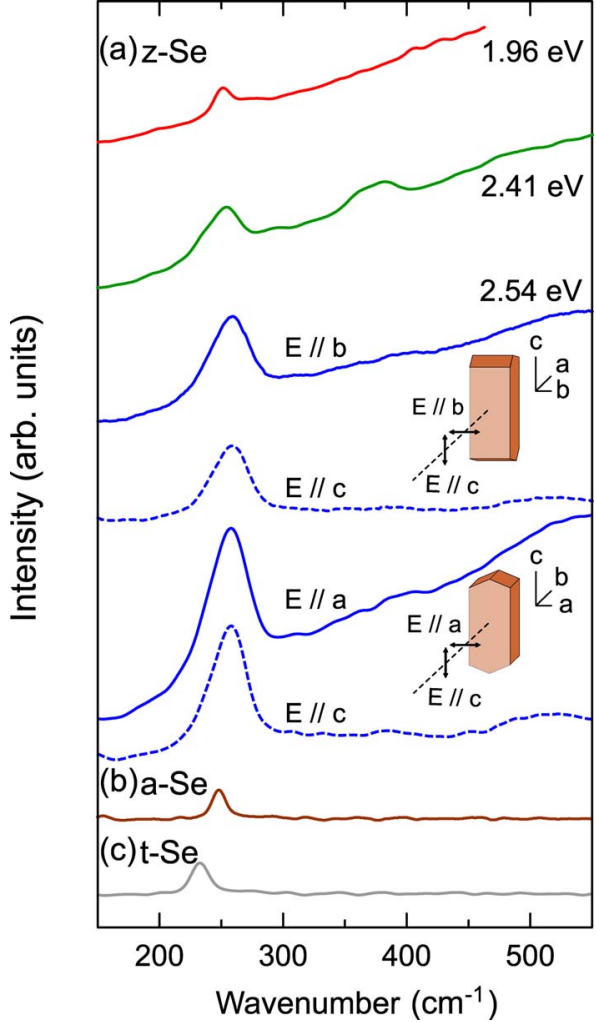

FIG. 2. (Color online) Raman-scattering spectra of (a) $z$-Se (six traces from the top), (b) $a$-Se, and (c) $t$-Se. For $z$-Se, the top and second spectra are obtained using probe light of $1.96 \mathrm{eV}(633 \mathrm{~nm})$ and $2.41 \mathrm{eV}(515 \mathrm{~nm})$ with polarization unresolved. All the others are probed by $2.54 \mathrm{eV}(488 \mathrm{~nm})$ light in which the electric field $E$ direction and incident plane $(a$ and $b)$ are illustrated in the insets.

\section{X-ray diffraction}

Figure 3 shows $x$-ray diffraction patterns obtained for assemblages of pure ZSM-5 and $z$-Se at scattering angles of $2 \theta=5^{\circ}-35^{\circ}$. At higher angles of up to $60^{\circ}$, there have been no discernible differences in the two profiles. We see in Fig. 3 that ZSM-5 shows Bragg peaks at $2 \theta \approx 8^{\circ}-10^{\circ}, 14^{\circ}$, and $23^{\circ}$, the pattern resembling that reported previously. ${ }^{37}$ With Se loading, these three peaks appear to be modified, which may reflect generated strains by the loading, as previously reported for S-loaded $\mathrm{Na} A{ }^{6}$ In addition, surprisingly, $z$-Se

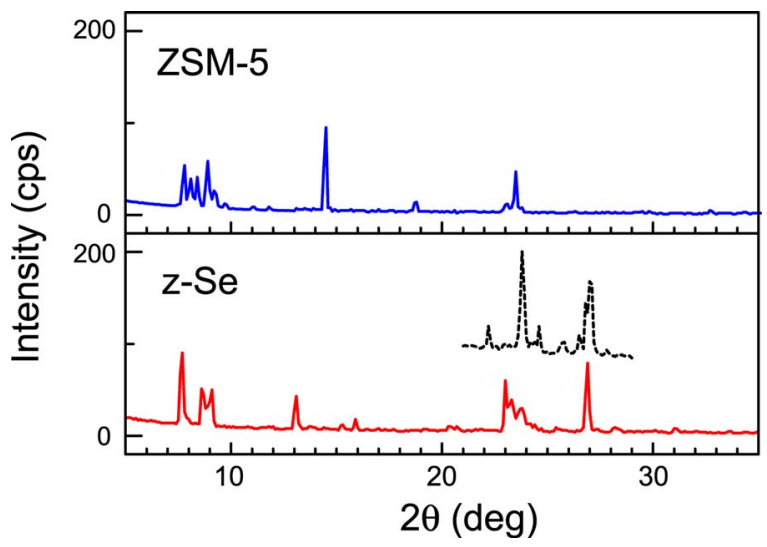

FIG. 3. (Color online) Transmission x-ray diffraction patterns of ZSM-5 and $z$-Se on highly oriented pyrolytic graphite substrates. The dashed black line in $z$-Se exemplifies sample-to-sample variation.

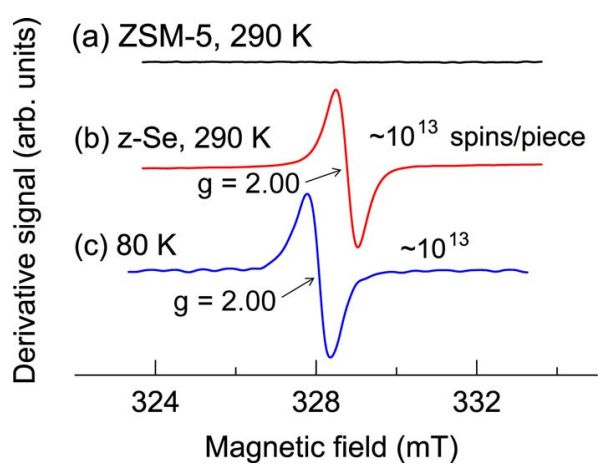

FIG. 4. (Color online) ESR spectra of (a) ZSM-5 at room temperature and $z$-Se at (b) room temperature and (c) $80 \mathrm{~K}$.

produces a clear peak at $\sim 27^{\circ}$, which indicates the existence of crystalline planes with a separation of $\sim 0.33 \mathrm{~nm}$. The peak width of $\sim 0.5^{\circ}$, which may be still influenced by the widths of diffractometer slits, suggests a crystal thickness of $\sim 80 \mathrm{~nm}$ through the Scherrer equation.

\section{E. ESR}

An assemblage ( $\sim 1000$ pieces) of $z$-Se has exhibited a strong and sharp ESR signal. As shown in Fig. 4(a), pure ZSM-5 and Se chunks (not shown), employed for sample preparation, have not presented any ESR signals. In contrast, $z$-Se exhibits a clear peak with the $g$-value and the half width of $\sim 2.00$ and $\sim 1 \mathrm{mT}(=10 \mathrm{G})$, which scarcely change with temperature at 80 and $290 \mathrm{~K}$. The signal strength, suggesting a spin density of $\sim 10^{13} /$ piece, also does not change with temperature. It is known that pure Se bulk samples produce detectable ESR signals at $g \simeq 2.00$ only under light irradiation at low temperatures, while the peak is much broader, 15-22 mT. ${ }^{40,41}$ Alternatively, it has been demonstrated that oxidized Se samples exhibit a sharp signal, the shape being very similar to the present one, which is ascribed to oxygen charge-transfer complexes, which terminate Se chains. ${ }^{39,42}$ The present ESR result is, to the authors' knowledge, the first demonstration of the oxygen complexes in Se-zeolite systems. A plausible reason of this finding is due to the covalency of ZSM-5, in which the interaction between Se chains and pore walls is weak. On the other hand, in previous studies, Se chains may be terminated by cation such as $\mathrm{Al}$ in the pore walls. Light irradiation effects on the present sample are reported in a following paper.

\section{F. Optical absorption}

Figure 5(a) shows optical absorption (attenuation) spectra of $z$-Se, in comparison to those of $t$-Se (Refs. 39, 43, and 44) and $a$-Se. ${ }^{39,45,46}$ As mentioned in Sec. II, $z$-Se exhibits substantial light scattering, and accordingly, the spectra have been evaluated in two ways. The solid line is calculated from the transmittance [Fig. 1(b)] obtained using the conventional spectrometer. On the other hand, the circles connected by a dashed line are obtained from measurements using lasers and photodetectors, which are located just after the sample, so that the spectrum is less influenced by light scattering. The 

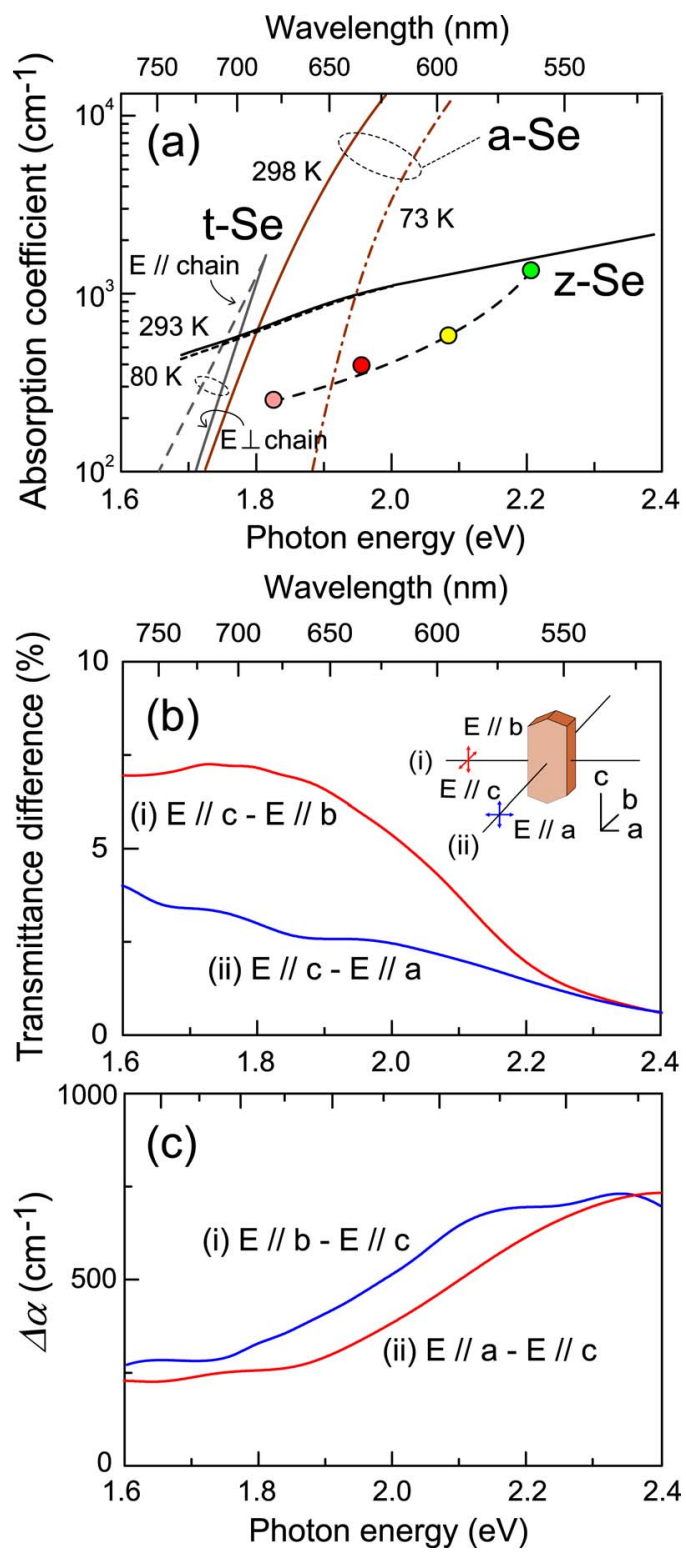

FIG. 5. (Color online) (a) Optical absorption (attenuation) and [(b) and (c)] dichroic spectra of $z$-Se. In (a), the solid line and the circles for $z$-Se are obtained using a conventional spectrometer and the laser measurement (polarization unresolved). Results for $t$-Se $(E \perp$ chain, $E \|$ chain) at room temperature (Refs. 39, 43, and 44) and for $a$-Se at 73 and $298 \mathrm{~K}$ (Ref. 45) are also shown for comparison. (b) shows anisotropic transmittance upon the direction of electric field $E$, the definition being shown in the inset. The absorption difference $\Delta \alpha$ in (c) is calculated from the spectra in (b).

two results seem to coincide above $\sim 2.2 \mathrm{eV}$. Spectra outside of 1.7-2.4 eV could not be evaluated due to the fixed shapes of the present samples.

We see that $z$-Se presents three marked features in comparison to the spectra of $a$-Se and $t$-Se. First, $z$-Se possesses a substantially gradual absorption edge, which is partially ascribable to light scattering. It also implies that loaded Se takes varied forms of clusters. Second, an optical gap energy, which may be defined as a photon energy at an absorption coefficient of $10^{3} \mathrm{~cm}^{-1}$, is located at $\sim 2.2 \mathrm{eV}$. It is blueshifted by $\sim 0.4 \mathrm{eV}$ from those of $\sim 1.8 \mathrm{eV}$ (Refs. 39 and 43-46) in $a$-Se and $t$-Se at room temperature. Last, the spectrum in $z$-Se appears to scarcely change with temperature, in marked contrast to the prominent temperature dependence in $a$-Se (Ref. 45) and $t$-Se (not shown). ${ }^{39,43}$ Note that this temperature independence, at least, at higher energies $(\geq 2.2 \mathrm{eV})$ cannot be ascribed to the light scattering but to an inherent absorption property.

In detail, as shown in Fig. 5(b), the optical transmittance of $z$-Se depends on the direction of electric field $E$. The transmittance of light with $E \| c$ is higher than those of $E \| a$ and $E \| b$, at least, at $\hbar_{\omega}=1.6-2.2 \mathrm{eV}$. On the other hand, transmittances of $E \| a$ and $E \| b$ cannot be compared directly since it is difficult to transmit light along the $c$-axis. Nevertheless, it has been reproducible in several samples that $E \| a$ has higher transmittance than that of $E \| b$. Summarizing these features, we assume the anisotropic absorbance, or dichroism, of $E\|b>E\| a>E \| c$.

\section{DISCUSSION}

The first problem is the form of Se clusters in the zeolite. Macroscopic observations have provided two insights. One is the inhomogeneous loading of Se, which is inferred from the spatial color variation of $z$-Se in Fig. 1. On the other hand, the weight-loss measurement suggests that the pores in ZSM-5 are mostly occupied by Se atoms.

The Raman-scattering spectroscopy manifests that the short-range structure of Se clusters in ZSM-5 is not very different from those in $t$-Se and $a$-Se. That is, since all the substances exhibit the main peaks at $240-260 \mathrm{~cm}^{-1}$, we envisage similar structures with the atomic coordination of two, nearest-neighbor distance of $\sim 0.23 \mathrm{~nm}$, and bond angle of $\sim 105^{\circ} .{ }^{39,47}$ We then assume Se chains or rings. However, ring molecules such as $\mathrm{Se}_{8}$, which is reported to give a peak at $\sim 270 \mathrm{~cm}^{-1}, 22$ are appreciably greater in size $(\sim 0.9 \mathrm{~nm})$ than the pore diameter in ZSM-5, ${ }^{35-37}$ and accordingly, the existence can be dismissed. For the chains, it is known that the position of the main Raman-scattering peak, arising from a symmetric bond stretching mode, shifts to higher wavenumbers with decreasing intermolecular interaction, from $236 \mathrm{~cm}^{-1}$ in $t$-Se to $\sim 260 \mathrm{~cm}^{-1}$ in isolated Se chains. ${ }^{22,39}$ This fact suggests that loaded Se in ZSM-5, showing the slightly higher peak position $\left(\sim 260 \mathrm{~cm}^{-1}\right)$ than those of $t$-Se and $a$-Se, takes chain forms with little interchain interaction, which is possibly single Se chains. On the other hand, the $380 \mathrm{~cm}^{-1}$ peak is assigned to a Se dimer, which is known to be stable in Se vapors. ${ }^{910,20,24,25}$ We therefore conclude that Se in ZSM-5 takes chain structures, having negligible interchain interaction, and dimers.

The chain length can be estimated. The weight-loss measurement has suggested the Se density of $\sim 10^{15}$ atom/piece, and the ESR shows a spin density of $\sim 10^{13} /$ piece, which is attributable to the oxygen charge-transfer complex, ${ }^{39,42}$ as mentioned in Sec. III. Accordingly, we envisage a single chain of $\sim 10^{2} \mathrm{Se}$ atoms, the ends being terminated by $\mathrm{O}_{2}$ molecules or $\mathrm{O}$ atoms. It is not known, however, whether the chain length distributes at $2-10^{2}$ or 2 and $\sim 10^{2}$.

Interpretation of $\mathrm{x}$-ray diffraction patterns is puzzling. Why does $z$-Se produce the sharp crystalline peak of $d$ $\approx 0.33 \mathrm{~nm}$ ? The distance is clearly longer than the first nearest-neighbor distance of $0.23 \mathrm{~nm}$ in Se solids and 
TABLE I. Comparison of threefold [Ref. 50] and fourfold helices having a bond length of $0.23 \mathrm{~nm}$ and a bond angle of $105^{\circ}$.

\begin{tabular}{lcccc}
\hline \hline Helix & $\begin{array}{c}\text { Rotating angle } \\
\left({ }^{\circ}\right)\end{array}$ & $\begin{array}{c}\text { Helix diameter (framework) } \\
(\mathrm{nm})\end{array}$ & $\begin{array}{c}\text { Helix pitch } \\
(\mathrm{nm})\end{array}$ & $\begin{array}{c}\text { Plane separation } \\
(\mathrm{nm})\end{array}$ \\
\hline Threefold & 120 & 0.19 & 0.50 & 0.22 \\
Fourfold & 90 & 0.28 & 0.74 & 0.37 \\
\hline \hline
\end{tabular}

slightly shorter than the second nearest-neighbor distance of $0.37 \mathrm{~nm} .{ }^{39}$ Otherwise, we notice that this separation is similar to that $(0.33 \mathrm{~nm})$ of crystalline 101 faces in $t-S e{ }^{47}$ However, it is doubtless that $t$-Se cannot geometrically exist in such narrow pores in ZSM-5. In addition, the existence of $t$-Se is also in conflict with the Raman peak position, as discussed above, and with the blueshifted and temperatureindependent optical absorption edge [Fig. 5(a)]. We then consider three other possibilities below.

First, it may be straightforward to assume a single threefold helical chain, illustrated in Fig. 6(a), which exists in $t$-Se. The chain has a framework helix diameter of $0.19 \mathrm{~nm}$ (Table I) (Ref. 48) so that the van der Waals diameter is estimated at $\sim 0.6 \mathrm{~nm},{ }^{49}$ which can geometrically exist in the zeolite pore with the framework and free diameters of $\sim 0.83$ and $\sim 0.56 \mathrm{~nm}$. In the threefold chain, trimers, Se$\mathrm{Se}-\mathrm{Se}$, fix small and parallel planes, the one being indicated as 1-3 in Fig. 6(a). A series of the parallel planes may give Bragg peaks. However, the plane separation is calculated to be $\sim 0.22 \mathrm{~nm}$, substantially smaller than the experimentally obtained distance of $\sim 0.33 \mathrm{~nm}$. Accordingly, we neglect this structure.

Second, we may assume a double threefold helical structure, shown in Fig. 6(b). As mentioned above, $t$-Se has threefold chains, and a closer inspection of the structure reveals that aligned two helices fix a plane, defined by 1-3 in Fig. 6(b). Moreover, a calculation manifests that the plane has a separation of $\sim 0.35 \mathrm{~nm}$, which is nearly the same as $d$ $\approx 0.33 \mathrm{~nm}$. Actually, Nozue et al. ${ }^{19}$ proposed on basis of optical results such double-chain structures for $\mathrm{Se}$ in $\mathrm{MgX}$ and $\mathrm{Na} Y$. However, the cross-sectional atomic distance of the structure, taking the van der Waals diameter of Se into account, is $\sim 0.9 \mathrm{~nm}$, which is clearly greater than the pore diameters in ZSM-5. In short, the double threefold helixes cannot exist.

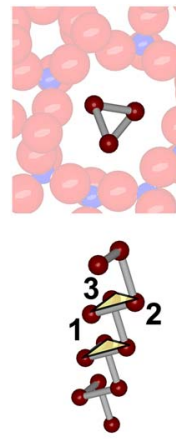

(a)
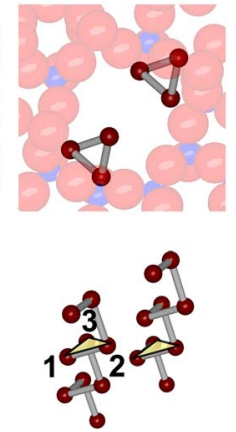

(b)
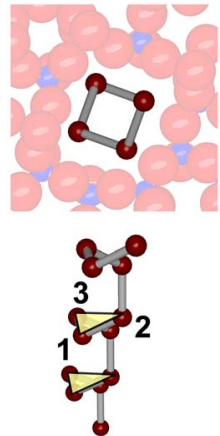

(c)
FIG. 6. (Color online) Cross-sectional (upper) and three-dimensional (lower) views of (a) single threefold, (b) double threefold, and (c) single fourfold helical chains in ZSM-5 pores.
Third, we propose a single fourfold helix, illustrated in Fig. 6(c). In such modified structures, however, the bond length $(\sim 0.23 \mathrm{~nm})$ and bond angle $\left(\sim 105^{\circ}\right)$ are retained due to big strain energies. A simple analysis under this condition shows that the fourfold helix has framework and van der Waals diameters of $0.28 \mathrm{~nm}$ and $\sim 0.68 \mathrm{~nm}$ (Table I), which are appreciably smaller than or comparable to the pore diameters of ZSM-5. In addition, the separation between the trimer plane [1-3 in Fig. 6(c)] is $\sim 0.37 \mathrm{~nm}$, nearly the same as $d \approx 0.33 \mathrm{~nm}$. This model is able to explain the position of the diffraction peak.

Previous studies may lend support to the stability of such chains. For instance, Katayama et al. ${ }^{18}$ experimentally demonstrated that Se in mordenite has a helix rotation angle of $104^{\circ}$, giving rise to 3.5 -fold helices. In theoretical calculations for Se chains in zeolites, Springborg and Jones ${ }^{50}$ and Ikawa and Fukutome ${ }^{51}$ suggested 3.3-fold and $\sim 4.5$-fold helices. It should be also noted that in the present case, since the sizes of the zeolite pore and the helix diameter are comparable, it is plausible that there exists small interaction between Se and the pore atoms, the number being even (2 $\times 10$ atoms). In addition, the pore shape appears to be rectangular. The pore may provide a matrix effect ${ }^{52}$ to the Se chain, modifying threefold to fourfold.

However, a remaining problem is if such single chains with small crystalline planes can give the narrow diffraction peak with a width of $\sim 0.5^{\circ}$. A single chain of $\sim 10^{2}$ atoms has a typical length of $\sim 20 \mathrm{~nm}$, while it is not evident if the segmental plane can give the sharp peak. We may speculate that several chains aligning in parallel pores in ZSM-5 (see, Fig. 1) cooperatively diffract the incident $\mathrm{x}$-ray beam, producing the sharp peak. To obtain reliable insight into the peak width, we need numerical calculations.

The blueshifted absorption edge [Fig. 5(a)] can be ascribed to the single chain structure. Theoretical calculations $^{51,53-55}$ demonstrate that the interchain interaction makes an energy width of the valence band, arising from the lone-pair electron state, broader by 1-2 eV. Accordingly, we expect that the single chain has a greater HOMO-LUMO gap by $\sim 1 \mathrm{eV}$ than the bandgap in $t$-Se, which is comparable to the observed blueshift of $\sim 0.4 \mathrm{eV}$. Otherwise, the shift can be understood as a one-dimensional quantum-well effect, which is estimated from $\Delta E=\left(\pi^{2} \hbar^{2} / 2 m\right) \Sigma_{i}\left(1 / L_{i}^{2}\right)$, where $m$ is a carrier mass and $L_{i}$ a well side length (or an electronic mean-free path if it is shorter). Provided $m$ to be the freeelectron mass and a rectangular well with a length of 1-20 $\mathrm{nm}$ and side lengths of $0.7 \mathrm{~nm}$ (van der Waals helix diameter), we also obtain $\Delta E \approx 1 \mathrm{eV}$. The very small temperature dependence of the absorption edge may imply weak 
electron-vibration coupling arising from nonexistence of interchain interaction, ${ }^{44}$ which is consistent with the single chain structure.

Finally, we consider the meaning of the optical anisotropy, probably suggesting a dichroic absorbance of $E \| b$ $>E\|a>E\| c$. Note that this sequence seems to correspond to the photoluminescence anisotropy, which is the weakest for $E \|_{c}$ (Fig. 2). To understand this optical anisotropy, we should know the absorptions of Se chains when $E$ is parallel ( $E \|$ chain) and perpendicular ( $E \perp$ chain) to the chain axis. For such purposes, however, experimental results for $t$-Se (Refs. 39, 43, and 44) give a confusing insight, in which the anisotropic absorption depends on the photon energy [see Fig. 5(b)] and samples. ${ }^{39,43,44}$ On the other hand, for single Se chains, there are at least two experimental reports on optical anisotropy, ${ }^{8,33}$ which are qualitatively consistent. Bogomolov et $a .^{8}{ }^{8}$ demonstrated for Se-loaded mordenite that $E \|$ chain gives a higher absorption. Li et al. ${ }^{33}$ demonstrated for Se-loaded AFI zeolites ${ }^{5}$ that single Se chains give an absorption peaked at $\sim 2.6 \mathrm{eV}$ only when $E \|$ chain. We then can assume that the $E \|$ chain configuration gives a higher absorption. In ZSM-5, on the other hand, the straight pore exists along the $b$-axis (Fig. 1), and it is plausible that $\mathrm{Se}$ chains more easily diffuse into such pores. Moreover, if single Se chains are located along the pore, the $E \| b$ configuration will give stronger absorption, which gives more intense photoluminescence (Fig. 2). The zig-zag pore, having a similar diameter, in the $a-c$ plane can also contain the single chain, which probably gives a contribution to absorption of $E\|a>E\|_{c}$ through projected chain lengths. Therefore, we expect $E\|b>E\| a>E \| c$ for the anisotropic absorbance, consistent with the experimental result in Fig. 5. Results on photoinduced phenomena and nonlinear optical properties of $z$-Se will be reported in a following paper (II) and elsewhere.

\section{CONCLUSIONS}

The atomic structure of Se in a covalent zeolite, submillimeter size ZSM-5 single crystals, has been investigated by means of weight measurement, Raman scattering, x-ray diffraction, ESR, and optical absorption spectroscopy. Loaded Se atoms appear to form single fourfold helical chains with atomic lengths of $\sim 10^{2}$ and dimers. The optical absorption edge blueshifts from those in $t$-Se and $a$-Se by $\sim 0.4 \mathrm{eV}$, which can be connected with the single chain structure. The optical anisotropy can be understood as manifestation of a greater cross section of single chains at $E \|$ chain and easier chain penetration into straight pores in ZSM-5.

\section{ACKNOWLEDGMENTS}

The authors would like to thank Dr. Y. Kiyozumi for supplying ZSM-5 samples, Dr. T. Hiraoki for ESR measurement, Dr. A. V. Kolobov and Dr. V. V. Poborchii for unpublished information, Dr. N. Terakado for photoluminescence measurement, and graduate students $\mathrm{S}$. Okamoto and $\mathrm{K}$. Noda for experimental assistance. The authors also acknowledge financial support from Nippon Sheet Glass Foundation for Material Science and Engineering.
${ }^{1} \mathrm{~K}$. Tanaka, in Encyclopedia of Nanoscience and Nanotechnology, edited by H. S. Nalwa (American Scientific, Valencia, 2004), p. 629.

${ }^{2}$ S. Kokenyesi, J. Optoelectron. Adv. Mater. 8, 2093 (2006).

${ }^{3}$ C. J. Zha, P. Osvath, G. Wilson, and A. Launikonis, Appl. Phys. A: Mater. Sci. Process. 94, 427 (2009).

${ }^{4}$ N. Terakado and K. Tanaka, Jpn. J. Appl. Phys. 47, 7972 (2008).

${ }^{5} \mathrm{http}: / /$ www.iza-structure.org/databases/

${ }^{6}$ S. A. Averkiev, L. S. Agroskin, V. G. Aleksandrov, V. N. Bogomolov, Y. N. Volgin, A. I. Gutman, T. B. Zhukova, V. P. Petranovskiî, D. S. Poloskin, L. P. Rautian, and S. V. Kholodkevich, Sov. Phys. Solid State 20, 251 (1978).

${ }^{7}$ V. N. Bogomolov, V. V. Poborchii, and S. V. Kholodkevich, JETP Lett. 31, 434 (1980).

${ }^{8}$ V. N. Bogomolov, S. V. Kholodkevich, S. G. Romanov, and L. S. Agroskin, Solid State Commun. 47, 181 (1983).

${ }^{9}$ A. Goldbach, L. Iton, M. Grimsditch, and M. L. Saboungi, J. Am. Chem. Soc. 118, 2004 (1996).

${ }^{10}$ A. Goldbach, M. Grimsditch, L. Iton, and M. L. Saboungi, J. Phys. Chem. B 101, 330 (1997)

${ }^{11}$ A. Goldbach, L. E. Iton, and M. L. Saboungi, Chem. Phys. Lett. 281, 69 (1997).

${ }^{12}$ L. He, Z. X. Shen, G. Gu, L. Qin, and S. H. Tang, Chem. Phys. Lett. 300, 504 (1999).

${ }^{13}$ A. A. Demkov and O. F. Sankey, J. Phys.: Condens. Matter 13, 10433 (2001).

${ }^{14}$ A. Goldbach, L. E. Iton, M. Grimsditch, and M. L. Saboungi, Chem. Mater. 16, 5107 (2004).

${ }^{15}$ K. Tamura, S. Hosokawa, H. Endo, S. Yamasaki, and H. Oyanagi, J. Phys. Soc. Jpn. 55, 528 (1986).

${ }^{16}$ O. Terasaki, K. Yamazaki, J. M. Thomas, T. Ohsuna, D. Watanabe, J. V. Sanders, and J. C. Barry, Nature (London) 330, 58 (1987).

${ }^{17}$ M. Inui, M. Yao, and H. Endo, J. Phys. Soc. Jpn. 57, 553 (1988).

${ }^{18}$ Y. Katayama, M. Yao, Y. Ajiro, M. Inui, and H. Endo, J. Phys. Soc. Jpn. 58, 1811 (1989).

${ }^{19}$ Y. Nozue, T. Kodaira, O. Terasaki, K. Yamazaki, T. Goto, D. Watanabe, and J. M. Thomas, J. Phys.: Condens. Matter 2, 5209 (1990).

${ }^{20}$ V. V. Poborchii, J. Phys. Chem. Solids 55, 737 (1994).

${ }^{21}$ K. Maruyama, T. Tsuzuki, M. Yao, and H. Endo, Surf. Rev. Lett. 3, 711 (1996).

${ }^{22}$ V. V. Poborchii, A. V. Kolobov, J. Caro, V. V. Zhuravlev, and K. Tanaka, Chem. Phys. Lett. 280, 17 (1997).

${ }^{23}$ Z. K. Tang, M. M. T. Loy, T. Goto, J. Chen, and R. Xu, Solid State Commun. 101, 333 (1997).

${ }^{24}$ S. Kohara, A. Goldbach, N. Koura, M. L. Saboungi, and L. A. Curtiss, Chem. Phys. Lett. 287, 282 (1998).

${ }^{25}$ A. V. Kolobov, H. Oyanagi, V. V. Poborchii, and K. Tanaka, Phys. Rev. B 59, 9035 (1999).

${ }^{26}$ V. V. Poborchii, A. V. Kolobov, J. Caro, V. V. Zhuravlev, and K. Tanaka, Phys. Rev. Lett. 82, 1955 (1999).

${ }^{27}$ K. Matsuishi, T. Isome, J. Ohmori, S. Onari, and T. Arai, Phys. Status Solidi B 215, 301 (1999).

${ }^{28}$ D. Reinen and G. G. Lindner, Chem. Soc. Rev. 28, 75 (1999).

${ }^{29}$ P. Simoncic and T. Armbruster, Microporous Mesoporous Mater. 71, 185 (2004).

${ }^{30}$ C. Bichara and R. J. M. Pellenq, Mol. Simul. 30, 781 (2004).

${ }^{31}$ I. L. Li, S. C. Ruan, Z. M. Li, J. P. Zhai, and Z. K. Tang, Appl. Phys. Lett. 87, 071902 (2005).

${ }^{32}$ I. L. Li, J. P. Zhai, P. Launois, S. C. Ruan, and Z. K. Tang, J. Am. Chem. Soc. 127, 16111 (2005).

${ }^{33}$ I. L. Li, P. Launois, and Z. K. Tang, Appl. Surf. Sci. 226, 36 (2004)

${ }^{34}$ A. Goldbach and M. L. Saboungi, Acc. Chem. Res. 38, 705 (2005).

${ }^{35}$ G. T. Kokotailo, S. L. Lawton, D. H. Olson, and W. M. Meier, Nature (London) 272, 437 (1978).

${ }^{36}$ H. von Koningsveld, J. C. Jansen, and H. V. Bekkum, Zeolites 10, 235 (1990).

${ }^{37}$ S. Shimizu and H. Hamada, Microporous Mesoporous Mater. 48, 39 (2001).

${ }^{38}$ A. Saitoh and K. Tanaka, Solid State Commun. 149, 750 (2009).

${ }^{39}$ Selenium, edited by R. A. Zingaro and W. C. Cooper (Van Nostrand Reinhold, New York, 1974).

${ }^{40}$ S. G. Bishop, U. Strom, and P. C. Taylor, Phys. Rev. Lett. 36, 543 (1976).

${ }^{41}$ A. V. Kolobov, M. Kondo, H. Oyanagi, A. Matsuda, and K. Tanaka, Phys. Rev. B 58, 12004 (1998). 
${ }^{42}$ M. Abkowitz, J. Chem. Phys. 46, 4537 (1967).

${ }^{43}$ W. Henrion, Phys. Status Solidi 20, K145 (1967).

${ }^{44}$ G. G. Roberts, S. Tutihasi, and R. C. Keezer, Phys. Rev. 166, 637 (1968).

${ }^{45}$ K. J. Siemens and E. W. Fenton, Phys. Rev. 161, 632 (1967).

${ }^{46}$ J. Stuke, J. Non-Cryst. Solids 4, 1 (1970).

${ }^{47}$ P. Andonov, J. Non-Cryst. Solids 47, 297 (1982).

${ }^{48}$ R. M. Martin and G. Lucovsky, Phys. Rev. B 13, 1383 (1976).

${ }^{49} \mathrm{~L}$. Pauling, The Nature of the Chemical Bond and the Structure of Molecules and Crystals: An Introduction to Modern Structural Chemistry
(Cornell University Press, New York, 1960).

${ }^{50}$ M. Springborg and R. O. Jones, J. Chem. Phys. 88, 2652 (1988).

${ }^{51}$ A. Ikawa and H. Fukutome, J. Phys. Soc. Jpn. 59, 1002 (1990).

${ }^{52}$ B. Smit and T. L. M. Maesen, Nature (London) 451, 671 (2008).

${ }^{53}$ I. Chen, Phys. Rev. B 7, 3672 (1973).

${ }^{54}$ J. D. Joannopoulos, M. Shluter, and M. L. Cohen, Phys. Rev. B 11, 2186 (1975).

${ }^{55}$ T. Yamaguchi and F. Yonezawa, J. Non-Cryst. Solids 156-158, 268 (1993). 